\title{
Flat Field Anomalies In An X-Ray CCD Camera Measured Using A Manson X-Ray Source
}

\author{
M. J. Haugh ${ }^{1}$, M. B. Schneider ${ }^{2}$ \\ ${ }^{1}$ National Security Technologies, PO Box 2710, Livermore, CA 94550 \\ ${ }^{2}$ Lawrence Livermore National Laboratory, PO Box 808, \\ Livermore, CA 94550 \\ 925/960-2538,haughmj@nv.doe.gov
}

\begin{abstract}
The Static X-ray Imager (SXI) is a diagnostic used at the National Ignition Facility (NIF) to measure the position of the X-rays produced by lasers hitting a gold foil target. The intensity distribution taken by the SXI camera during a NIF shot is used to determine how accurately NIF can aim laser beams. This is critical to proper NIF operation. Imagers are located at the top and the bottom of the NIF target chamber. The CCD chip is an X-ray sensitive silicon sensor, with a large format array (2k x $2 \mathrm{k}), 24 \mu \mathrm{m}$ square pixels, and $15 \mu \mathrm{m}$ thick. A multi-anode Manson X-ray source, operating up to $10 \mathrm{kV}$ and $10 \mathrm{~W}$, was used to characterize and calibrate the imagers. The output beam is heavily filtered to narrow the spectral beam width, giving a typical resolution $\mathrm{E} / \Delta \mathrm{E} \approx 10$. The $\mathrm{X}$-ray beam intensity was measured using an absolute photodiode that has accuracy better than $1 \%$ up to the Si K edge and better than $5 \%$ at higher energies. The $\mathrm{X}$-ray beam provides full CCD illumination and is flat, within $\pm 1 \%$ maximum to minimum. The spectral efficiency was measured at 10 energy bands ranging from $930 \mathrm{eV}$ to $8470 \mathrm{eV}$. We observed an energy dependent pixel sensitivity variation that showed continuous change over a large portion of the CCD. The maximum sensitivity variation occurred at $8470 \mathrm{eV}$. The geometric pattern did not change at lower energies, but the maximum contrast decreased and was not observable below $4 \mathrm{keV}$. We were also able to observe debris, damage, and surface defects on the CCD chip. The Manson source is a powerful tool for characterizing the imaging errors of an X-ray CCD imager. These errors are quite different from those found in a visible CCD imager.
\end{abstract}

\subsection{Introduction}

X-ray sensitive CCD cameras are often needed to provide quantitative intensity information for experimental studies in plasma physics and astronomy diagnostics. They must be characterized and calibrated in order to provide reliable analysis. ${ }^{1,2}$ We have used a multi-anode Manson X-ray source to calibrate X-ray CCD cameras used on the NIF target chamber. The X-ray beam is filtered so that spectral content includes only the $\mathrm{K}$ or $\mathrm{L}$ lines of the anode and the bremmstrahlung lying between them. The CCD Quantum Efficiency shows a similar pattern to that observed by others. ${ }^{3}$ The CCD showed two types of large deviations in sensitivity, both photon energy dependent. One pattern was prominent at high photon energies and decreased in amplitude as the photon energy decreased from the highest photon energy $(8470 \mathrm{eV})$ used and disappeared by $3000 \mathrm{eV}$. The other pattern was maximum amplitude at the lowest photon energy used and decreased as the photon energy increased until it disappeared by $4500 \mathrm{eV}$.

\subsection{SXI operation and CCD camera description}

The Static X-ray Imager (SXI) is a diagnostic used on the National Ignition Facility (NIF) at the Lawrence Livermore National Laboratory. The intensity distribution taken by the SXI camera during a NIF shot is used to determine how accurately NIF can aim laser beams. Critical to proper NIF operation, SXI diagnostics are located at both the top and the bottom of the NIF target chamber. The SXI CCD camera is X-ray sensitive, using a silicon sensor that is thinned and back illuminated. The silicon pixel array comprises a $2 \mathrm{k}$ x $2 \mathrm{k}$ array, $24 \mu \mathrm{m}$ square pixels, $15 \mu \mathrm{m}$ thick.

This work was done by National Security Technologies, LLC, under Contract No. DE-AC52-06NA25946 with the U.S. Department of Energy. DOE/NV/25946--455. This work was done under the auspices of the U.S. Department of Energy by Lawrence Livermore National Laboratory under Contract DE-AC52-07NA27344. 


\subsection{Experimental Method}

\subsection{Manson System Operating Principles}

The NSTec Manson type X-ray source provides X-rays in the energy range from $100 \mathrm{eV}$ to 9 $\mathrm{keV}$. These X-rays are used for various testing and calibration functions. As shown in the schematic diagram in Figure 2, the Manson comprises three compartments: the source chamber and two testing chambers. The test chambers are the rectangular boxes in the figure. The two test chambers are connected to the main chamber by stainless steel vacuum components that include an isolation gate valve and a mechanical shutter.

The Manson system is a multi-anode device because it can hold up to six different anodes on a hexagonal mounting bracket. Anodes are changed by rotating the bracket. The filament sitting in front of the anode produces a spot size of approximately $1 \mathrm{~mm}$ diameter circle. Two X-ray beams are isolated from the anode emission for use in the test chambers.

Each test chamber has its own vacuum pump and controls. A test chamber can be isolated from the source chamber by the gate valve and brought to atmosphere. Each test chamber holds a photodiode and an energy dispersive detector for measuring the X-ray flux and the $\mathrm{X}$-ray spectrum. The detectors are mounted on push rods so that they can be moved into or out of the beam. Items for calibration can be mounted in the chamber or attached to the rear of the chamber, as shown in Figure 2 and noted as "Diagnostic."

The X-ray beam paths that are used for testing are shown in Figure 2. Filter 1 shown in the source chamber is used to isolate a narrow wavelength band of X-rays. These filters are mounted in a vertical stalk that holds up to three filters. A light blocker prevents visible light from entering the test chamber. Visible light is emitted by the hot source filament and would overwhelm the detectors and CCD.

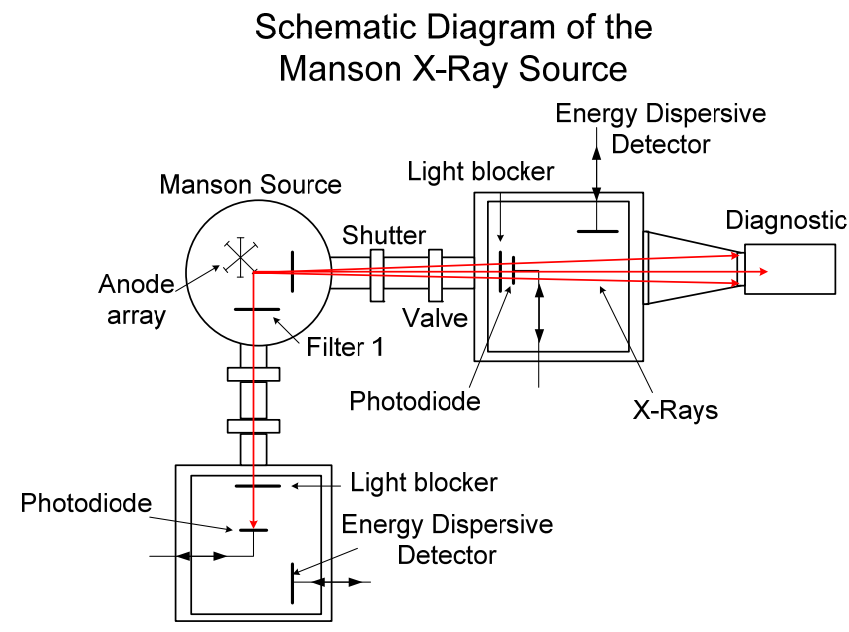

Figure 1. Manson Schematic; the SXI camera CCD is mounted 27.5 in from the rear of the reference box.

\subsection{Energies and Bandwidths}

The typical spectrum of the anode emission is broad band and includes bremsstrahlung and spectral lines of the anode material. The spectrum obtained from a Ti anode with the source at 10 $\mathrm{kV}$ is shown in Figure 3, which shows a spectrum produced by the Amptek energy dispersive detector. This detector has a resolution near $240 \mathrm{eV}$ FWHM. The Ti K lines are seen at $4500 \mathrm{eV}$ and $4900 \mathrm{eV}$. The actual Ti spectrum that we would see using a high resolution spectrometer has a natural spectral line width of about $2 \mathrm{eV}$ for these 3 emission lines. The bremsstrahlung can be 
estimated from the spectrum shown in Figure 3 and the $100 \mu \mathrm{m}$ thick Ti filter transmission values taken from the CXRO web site. ${ }^{4}$ An actual spectrum obtained from an Amptek detector when a $100 \mu \mathrm{m}$ Ti filter was in place is shown in Figure 3 with the unfiltered spectrum. Now the bandwidth of the radiation is considerably reduced to a bandwidth of nearly $450 \mathrm{eV}$. This thickness is a practical trade off between a narrow bandwidth and sufficient photon intensity to calibrate the camera.

All spectral band widths used for the SXI calibration were obtained in a similar manner. A filter having the same material as the anode was used. The band widths are all given by $\mathrm{E} / \Delta \mathrm{E} \sim 10$. Eleven spectral bands were used from $930 \mathrm{eV}$ to $8470 \mathrm{eV}$.

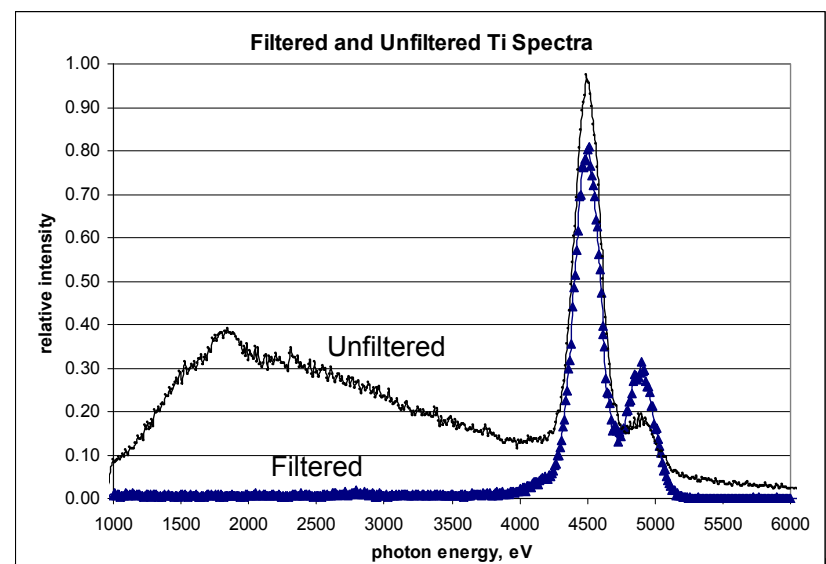

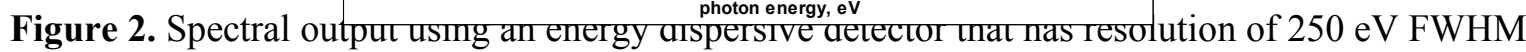
for a titanium anode, with filter and without filter.

\subsection{Camera pixel count relation to energy}

The SXI diagnostic needs to quantify the X-ray intensity coming from each region of the target. The SXI camera user needs to how the pixel signal, $\mathrm{S}(\mathrm{E})$ in counts per pixel, is related to the photon energy, E. A useful model is: ${ }^{6}$

$$
S=P \bullet A_{p i x} \bullet \tau \bullet Q E \bullet \eta \bullet\left(\frac{1}{K}\right)
$$

Where $\mathrm{P}$ is the photon rate, $\mathrm{A}_{\text {pix }}$ is the pixel area, $\tau$ is the exposure time, QE (quantum efficiency) is the fraction of photons that interact with the pixel, $\eta$ (quantum yield) is the number of electron-hole pairs produced by the photon, and $\mathrm{K}$ is the camera gain (counts per electron-hole pair). The camera manufacturer's measurement of the gain constant was used for the calculations. It was checked using visible light for one camera.

Photon intensity was measured using a photodiode. We use a photodiode manufactured by International Radiation Detectors, Inc. (IRD), model AXUV100. These detectors are designed with no doped dead region and zero surface recombination, so that they have near theoretical quantum efficiencies for the soft X-ray spectral region. One unit of this model was sent for calibration to the synchrotron at Physikalische-Technische Bundesanstalt (PTB). ${ }^{5}$ The results from this calibration showed $1 \%$ agreement from $1000 \mathrm{eV}$ to $1839 \mathrm{eV}$ and the photodiode gave $5 \%$ lower reading above this energy. The thickness of the Si photodiode is $54.5 \mu \mathrm{m}$.

The X-ray photon interacts with the Si sensor to produce hole-electron pairs that the CCD electronics process to produce the digital signal count $S$. The number of electron-hole pairs produced by an $\mathrm{X}$ - 
ray photon that interacts with the Si sensor is a function of the photon energy and is slightly dependent upon the temperature. ${ }^{7}$ The sensor is cooled to $253 \mathrm{~K}$ when operating. We used a value of $\eta=E / 3.66$ for the camera and $\eta=E / 3.62$ for the photodiode operating at room temperature.

\subsection{Methods for Imaging}

The X-ray beam filled the CCD. The camera linear range was determined first. The cameras had a large number of bad rows and hot pixels. The bad rows were associated with the readout and identified using closed shutter images with $3 \mathrm{~ms}$ exposure time. The hot pixels were identified by taking an image using the Ti anode and no filter and using the same exposure time that was used for the experiments on the NIF target chamber experiments. A map was made identifying the bad rows and bad pixels.

The photon intensity was measured with the photodiode in arm \#1. An exposure time was chosen to be as short as possible to give a reasonable signal. Photodiode readings were taken before and after each image. During imaging, the X-ray beam intensity was monitored continuously for beam fluctuations using the photodiode in arm \#2. If there were beam intensity fluctuations observed during imaging, that image was discarded. Four images were taken at each photon energy.

Flat field images were taken using the same anode voltage that was used for the camera efficiency measurements and maximum anode current. The exposure time was chosen to produce a signal that was $50 \%$ to $60 \%$ of saturation. Ten flat field images and ten background images were taken at each photon energy.

\subsection{Image Analysis}

The camera images for the efficiency analysis had the background subtracted and the bad pixels replaced by the average of adjacent pixels. The mean pixel count was determined by randomly selecting 1000 regions 20x20 pixels in size, calculating the mean counts/pixel for each region and calculating the average of the region means. This is the signal $S$ for that image. For the flat field images, average all images that have the same exposure time, average the background images and subtract the average background from the average flat field image.

\subsection{Results}

\subsection{Camera spillover}

Several images were taken using a low X-ray intensity and short exposure times so that individual photon results could be observed. These images show that for a majority of the single photon events, the X-ray energy spills over into adjacent pixels even for $24 \mu \mathrm{m}$ square pixels.

\subsection{Camera efficiency}

The camera efficiency for one of the SXI cameras is given in Figure 3.

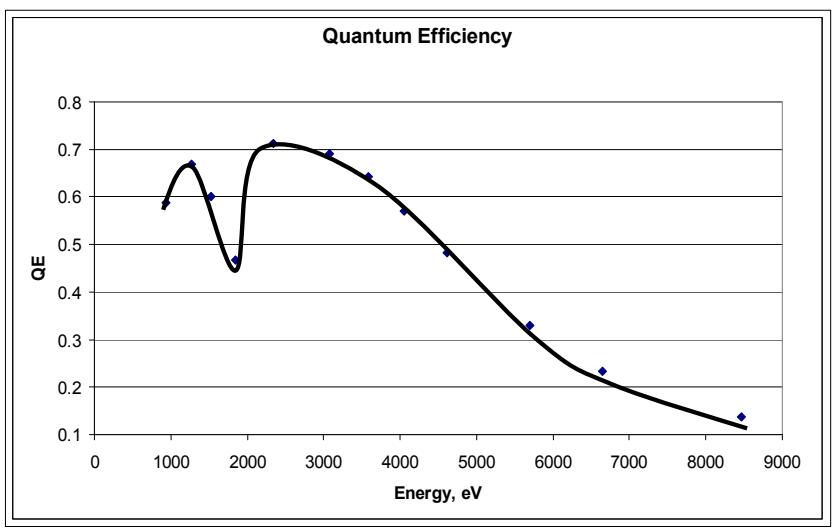


Figure 3. An SXI camera quantum efficiency plotted as a function of X-ray energy.

The Quantum Efficiency $(Q E)$ calculated using equations 3 and 5 and $\mathrm{K}=7.62$ electrons per count is plotted as a function of photon energy in Figure 3. The data scatter as measured by the standard deviation was $1 \%$ or less at each point. The dip near $1800 \mathrm{eV}$ and the fall-off after $2000 \mathrm{eV}$ are properties of $\mathrm{Si}$. Si that is $15 \mu \mathrm{m}$ thick transmits up to $35 \%$ as it approaches the K edge at $1839 \mathrm{eV}$. It begins transmitting again above $2500 \mathrm{eV}$ and is transmitting $80 \%$ at $8 \mathrm{keV}$. These $Q E$ results are similar to that obtained by Poletto. ${ }^{3}$ The line in the graph follows the data points except at the region before the K edge where it was adjusted to match the Si transmission curve. A point was added at $1837 \mathrm{eV}$ that was estimated by using the Si transmittance at $1837 \mathrm{eV}$ and the QE measured at 2345 $\mathrm{eV}$. There are two possible causes why the $Q E$ does not approach 1 when the photons are completely absorbed: (1) There may be absorption at the surface coating of the $\mathrm{Si}$; (2) the Quantum Yield may be less than the photon energy divided by $3.66 \mathrm{eV}$ per electron/hole pair. Analysis of a large number of single photon events could show the relative contribution of each effect.

\subsection{Flat Field}

The flat field source is the $1 \mathrm{~mm}$ diameter spot on the anode. The anode is $1405 \mathrm{~mm}$ from the CCD. This arrangement would produce a flat field to better than $1 \%$ if there were nothing between the anode and the CCD. There is a light blocker that has an aluminum coating on a polyimide film (Al $1054 \AA \pm 50 \AA$; polyimide $1081 \AA \pm 100 \AA$ ). This item does not affect the flat field within the $1 \%$ cited above. The filter can cause a variation in the beam intensity across the CCD if there is sufficient variation in thickness, foreign material, or misalignment with the anode. A comparison of all the flat field images implies that the maximum variation is $\pm 1 \%$ peak-to-peak.

Figure 7 shows the flat field image for one of the SXI cameras at the $\mathrm{Cu} 8470 \mathrm{eV}$ energy band. The image is set at high contrast so that the pixel signal variation shows clearly. A gross pattern is observed with the sensitivity at a maximum near the left center and decreasing slowly going away from the maximum. The image in Figure 8 is at $\mathrm{Ti} 4620 \mathrm{eV}$; it shows the same pattern but decreased magnitude. The pattern continues to decrease in magnitude until it is no longer visible at $3000 \mathrm{eV}$. Cross sections were calculated for 3 energies using the rectangular area shown in Figures 7 and 8. The computed cross sections are shown in the graph of Figure 9, given as the relative counts normalized to the maximum count and plotted as a function of the vertical (Y) pixel. The maximum sensitivity variation for each of the curves in Figure 9 is $13 \%$ at $8470 \mathrm{eV}, 6 \%$ at $4620 \mathrm{eV}$ and $2 \%$ at $3580 \mathrm{eV}$.

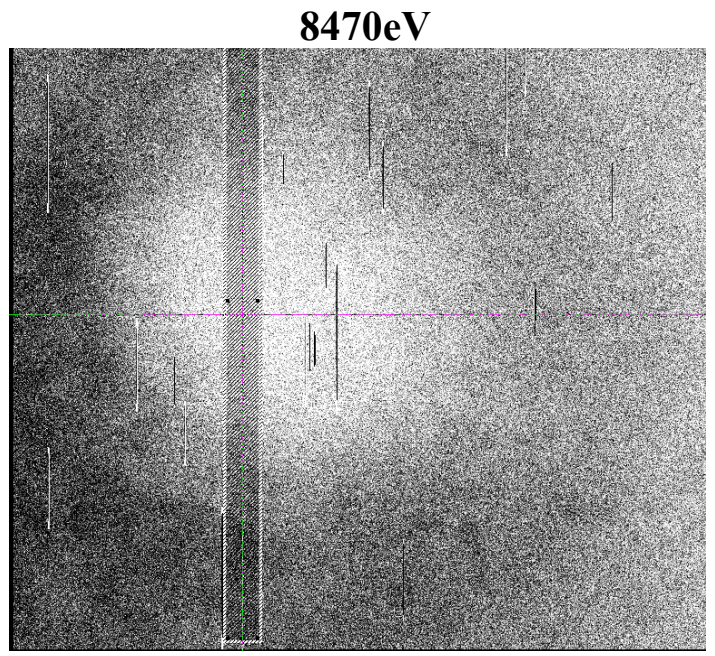

Figure 4. Flat field image for the $\mathrm{Cu}$ anode, $8470 \mathrm{eV}$ showing the pixel sensitivity variation. The vertical rectangle was the area used to calculate the cross section that is shown in Figure 11. (Signal range: 5200 to 7200 counts/pixel) 


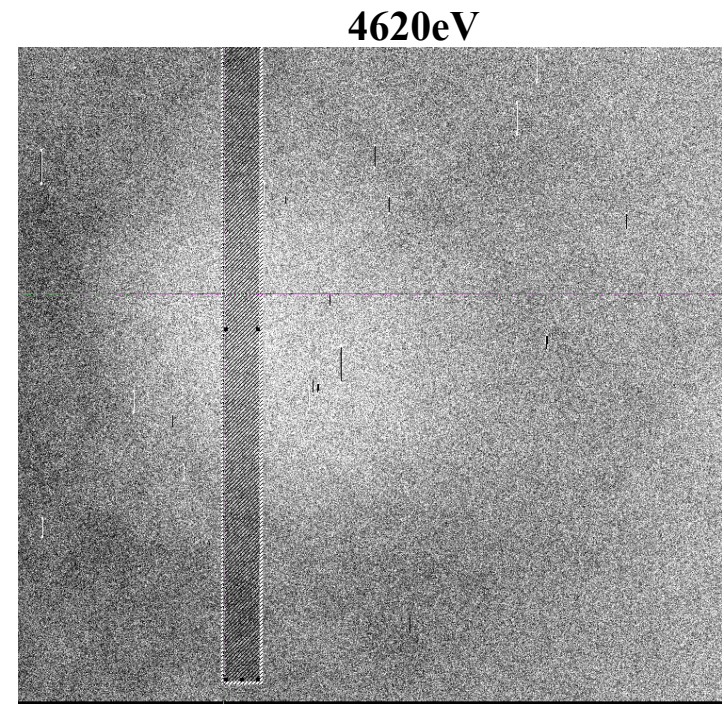

Figure 5. Flat field image for the Ti anode, $4620 \mathrm{eV}$ showing the pixel sensitivity variation. The vertical rectangle was the area used to calculate the cross section that is shown in Figure 11. (Signal range: 5200 to 7200 counts/pixel)

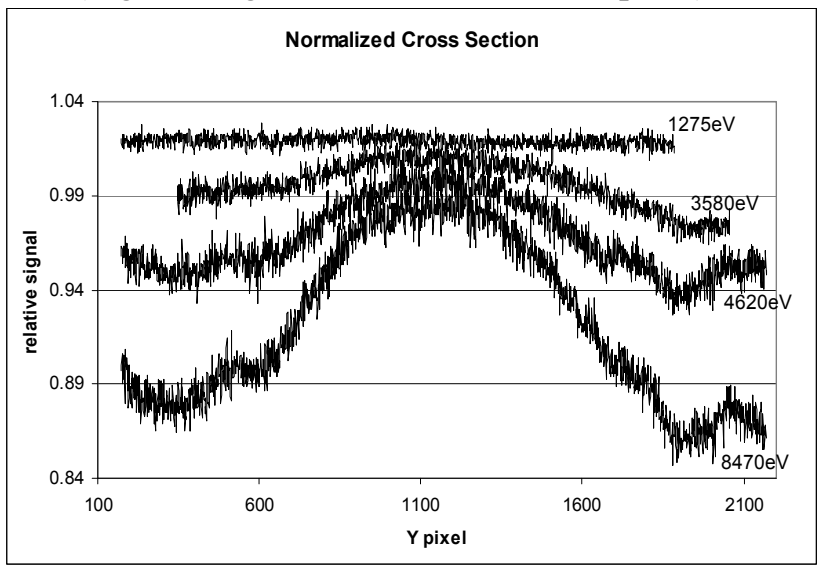

Figure 6. Relative cross sections for the area shown on the vertical rectangle in the images. These curves show how the magnitude of the pixel sensitivity decreases as the energy decreases.

A flat field image of the $\mathrm{Mg} 1275$ band is shown in Figure 10 for comparison to the higher energy flat field images. There is no trace of the sensitivity variation pattern that is seen at higher energies. A graph of the relative cross section at this energy and at the same location is also shown in figure 9. The $1275 \mathrm{eV}$ data on the graph shows that the maximum variation is less than $1 \%$, which is the measurement limit of our flat field procedure.

This sensitivity variation is a large scale effect; it includes groups of pixels and is probably related to the CCD manufacturing process. Any sensitivity variation of individual pixels is less than the photon noise associated with averaging 10 images. 


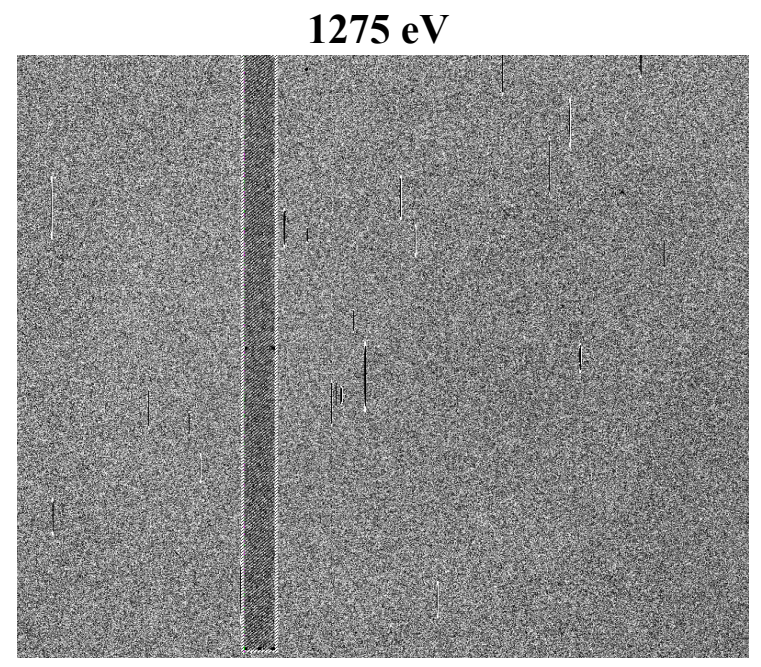

Figure 7. Flat field image for the $\mathrm{Mg}$ anode, $1275 \mathrm{eV}$, showing the pattern observed at the higher energies shown in Figures 7 and 8 has completely gone and the pixel sensitivity is flat. The vertical rectangle was the area used to calculate the cross section that is shown in Figure 9.

A different phenomenon was seen at low energies. Small irregular patches having diminished sensitivity were observed that are readily seen in Figure 11. This image shows a portion of the CCD. The effect on sensitivity in these regions also shows an energy dependence. Figure 12 is a similar image taken at $3080 \mathrm{eV}$. The irregular patches have now become quite dim compared to what was observed at $1275 \mathrm{eV}$. At $4500 \mathrm{eV}$, these paths of low sensitivity have completely disappeared.

$1275 \mathrm{eV}$

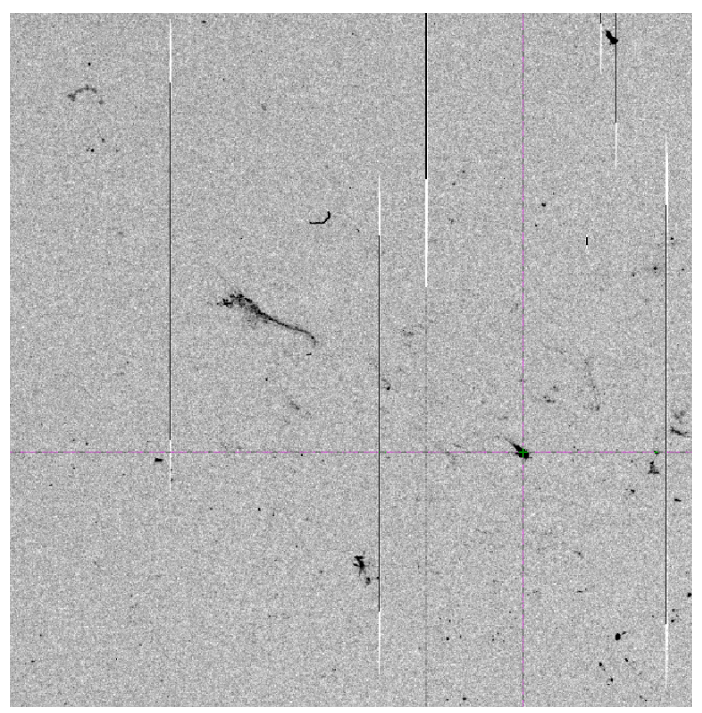

$3080 \mathrm{eV}$

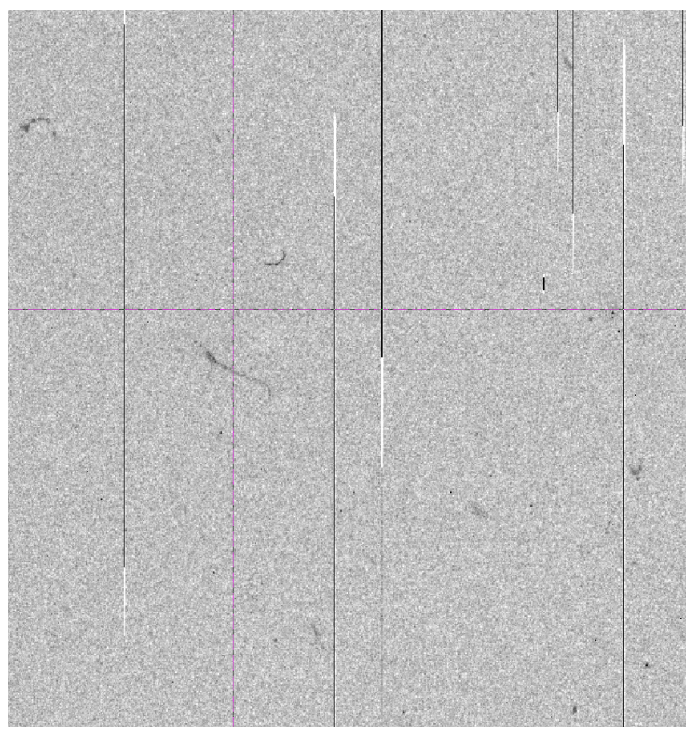

Figure 8. These are the same sections of a flat field image taken at 2 different energies, $1275 \mathrm{eV}$ and $3080 \mathrm{eV}$. The sections cover about $1 / 4$ of the entire CCD. The dark regions are CCD surface defects causing diminished pixel sensitivity. For the $1275 \mathrm{eV}$ section shown in $8 \mathrm{a}$ the blemishes are much darker than the $3080 \mathrm{eV}$ image shown in $8 \mathrm{~b}$.

There are several possible causes for these dark regions. Debris on the CCD surface could absorb Xrays and would be energy dependent, absorbing X-rays less as the energy increased. Damage to the 
CCD Would likely cause an energy dependence that would increase the variance of the defective region from the surrounding pixels as the energy increased. Damage to the surface coating could produce this effect if the coating were thicker in that defective region. When we examined the CCD surface with a magnifying glass it did appear that the coating was deformed. It looked like a manufacturing defect.

It is difficult to correct these images using the normal method of flat field inversion. This could be done if you limit the energy range of the X-ray source. But the characterization always provides the camera user with information that allows him to use the X-ray camera more effectively.

\subsection{Conclusions}

A Manson type source with proper filtering is a powerful tool for calibrating and characterizing the performance of a CCD camera in the energy range from $400 \mathrm{eV}$ to $9000 \mathrm{eV}$. CCD sensors can have defects that are large scale and energy dependent. A localized Quantum Efficiency measurement will not find these conditions. It is necessary to view the entire CCD with a flat source of X-rays.

\subsection{References}

1. Nakajima, H., Yamaguchi, H., Matsumoto, T., Tsuru, T., Koyama, K., Kissel, S., LaMarr, B., and Bautz, M., "Ground Calibration of CCD Cameras (XIS) with Front Illuminated Chips Onboard Astro-E2," Nuclear Instruments and Methods in Physics Research Section A 541, p365 (2005).

2. LaMarr, B., Bautz, M., Kissel, S., Prigozhin, G., Hayashida, K., Tsuru, T., and Matsumoto, H., "Ground Calibration of CCD Camera with Charge Injection for the X-ray Imaging Spectrometer on Astro-E2," Proc SPIE 5488, 124-135 (2004).

3. Poletto, L., Boscolo, A., and Tondello, G., "Characterization of a Charge-coupled Detector in the $1100-0.14 \mathrm{~nm}(1 \mathrm{eV}$ to $9 \mathrm{keV})$ Spectral Range," APPLIED OPTICS, $\underline{38}, 1$ Jan 99.

4. Center for X-ray Optics, Lawrence Berkeley Laboratory, http://www-cxro.lbl.gov .

5. Gottwald, A., Kruth, U., Richter, M., Scholze, F., and Ulm, G., Metrologia 43, S125, (2006).

6. Janesick, James R., Scientific Charge-Coupled Devices, SPIE Press, Bellingham, WA, 2001.

7. Knoll, Glenn F., Radiation Detection and Measurement, third edition, John Wiley \& Sons, 2000 . 